\title{
PAPER
}

\section{Infants' goal anticipation during failed and successful reaching actions}

\section{Amanda C. Brandone, ${ }^{1}$ Suzanne R. Horwitz, ${ }^{2}$ Richard N. Aslin ${ }^{3}$ and Henry M. Wellman ${ }^{4}$}

1. Department of Psychology, Lehigh University, USA

2. Department of Psychology, Yale University, USA

3. Department of Brain and Cognitive Sciences, University of Rochester, USA

4. Department of Psychology, University of Michigan, USA

\begin{abstract}
The ability to interpret and predict the actions of others is crucial to social interaction and to social, cognitive, and linguistic development. The current study provided a strong test of this predictive ability by assessing (1) whether infants are capable of prospectively processing actions that fail to achieve their intended outcome, and (2) how infants respond to events in which their initial predictions are not confirmed. Using eye tracking, 8-month-olds, 10-month-olds, and adults watched an actor repeatedly reach over a barrier to either successfully or unsuccessfully retrieve a ball. Ten-month-olds and adults produced anticipatory looks to the ball, even when the action was unsuccessful and the actor never achieved his goal. Moreover, they revised their initial predictions in response to accumulating evidence of the actor's failure. Eight-month-olds showed anticipatory looking only after seeing the actor successfully grasp and retrieve the ball. Results support a flexible, prospective social information processing ability that emerges during the first year of life.
\end{abstract}

\section{Introduction}

The ability to understand and predict the intentions of others' actions represents a major developmental achievement that is crucial to social interaction as well as to social, cognitive, and linguistic development (e.g. Baldwin \& Moses, 2001; Csibra \& Gergely, 2007; Tomasello, 1999). Research suggests that this cornerstone of social cognition has its roots in infancy. In the first year of life, infants are sensitive to the intentional structure of human actions and readily interpret others' actions as rational and goal directed (Baldwin, Baird, Saylor \& Clark, 2001; Brandone \& Wellman, 2009; Gergely, Nadasdy, Csibra \& Biro, 1995; Phillips \& Wellman, 2005; Woodward, 1998). Most existing research has examined these abilities using habituation techniques and the resulting looking-time data reflect retrospective judgments that occur after the action is completed. However, recently, researchers have begun investigating whether infants can also infer an actor's goal before it is achieved and then use that goal information to understand and predict actions as they unfold over time. For adults such a prospective intentional stance is fundamental to interpreting actions in real-time social situations and thus to interacting seamlessly with others. In this paper we examine infants' ability to respond flexibly and prospectively to complex social events involving unsuccessful intentional actions.

To examine whether infants can generate predictions about an actor's goal as it is unfolding over time, researchers have utilized eye-tracking methodology. Eye tracking enables researchers to examine infants' prospective judgments by measuring anticipatory looking patterns (i.e. whether participants look to the goal of an action before the action is completed). Several recent studies have shown that infants indeed generate anticipatory gaze shifts when they observe others engaging in successful, functional actions which the infants 
themselves are able to produce (Cannon \& Woodward, 2012; Falck-Ytter, Gredebäck \& von Hofsten, 2006; Gredebäck \& Melinder, 2010; Gredebäck, Stasieweicz, Falck-Ytter, Rosander \& von Hofsten, 2009; Hunnius \& Bekkering, 2010; Kanakogi \& Itakura, 2011; Kochukhova \& Gredebäck, 2010).

For example, Falck-Ytter and colleagues (2006) showed 6-month-olds, 12-month-olds, and adults videos in which an actor placed toys inside a bucket or sat passively as the toys moved to the bucket independently. The crucial measure was when participants first looked to the bucket and whether they did so before the toys arrived there. When the actor moved the toys, 12-montholds anticipated the goal of the action (much as adults did). In contrast, 6-month-olds failed to do so; they reactively shifted their gaze to the bucket only after the toy arrived there. Additional findings suggest that even 6-month-olds can anticipate simple, familiar actions that occur frequently in infants' own experience (e.g. a grasping hand reaching an object, Kanakogi \& Itakura, 2011; the arrival of a spoon or a cup to an actor's mouth during a feeding or drinking event, Kochukhova \& Gredebäck, 2010; Hunnius \& Bekkering, 2010). These data suggest that during the first year of life, infants can evaluate intentional human actions prospectively to the extent that they anticipate regularities in familiar goaldirected actions.

Evidence from Cannon and Woodward (2012) further demonstrates that infants can make predictions about the goals and not simply the physical endpoints of intentional human actions. In their study, 11-month-olds were familiarized with a hand repeatedly reaching to grasp one of two toys. The locations of the toys were then reversed and infants' anticipatory looks were measured. Results showed that infants looked with anticipation to the familiarized object (i.e. the goal) rather than to the familiarized location (i.e. the physical endpoint) of the action. These data demonstrate that when given prior information about an agent's goal, infants can predict the goal of a subsequent reaching action before the goal is achieved.

Together these studies provide firm evidence in support of infants' ability to generate predictions about others' actions as they unfold over time. Crucially, however, research to date has only assessed young infants' goal prediction abilities in situations in which the actor's goals are successfully completed (e.g. the actor grasps the desired object or brings the spoon to the person's mouth) and infants' initial expectations about the outcome of the action are confirmed (see Senju, Southgate, Snape, Leonard \& Csibra, 2011; Southgate, Senju \& Csibra, 2007, for evidence of older toddlers' ability to generate predictions about more complex social events). The reality of the social world and of intentional action is far more complicated than this. Many everyday acts are unsuccessful, interrupted, incomplete, or only partly observable. Moreover, in typical social interactions one's predictions about what a person is going to do next are often incorrect and must be revised in response to accumulating information. Consider the case of a mother who is in the process of reaching for a bottle to give to her infant when she accidentally knocks the bottle off the table or stops mid-reach to answer the phone. Mature social information processing requires the ability to respond flexibly and prospectively even in the case of these more complex events. Thus, a stronger test of infants' action prediction abilities would assess (1) whether infants are capable of prospectively processing actions that fail to achieve their intended outcome, and (2) how infants respond to events in which their initial goal predictions are not confirmed.

Several recent studies have demonstrated that infants in the first year of life can interpret incomplete or failed actions in terms of their unseen goals (Behne, Carpenter, Call \& Tomasello, 2005; Daum, Prinz \& Aschersleben, 2008; Hamlin, Hallinan \& Woodward, 2009; Hamlin, Newman \& Wynn, 2009; Legerstee \& Markova, 2008; Southgate \& Csibra, 2009). For example, Behne and colleagues (2005) examined infants' reactions to incomplete actions in the context of a toy-sharing situation. They asked whether infants would respond differently when an experimenter tried but failed to give them a toy (e.g. she accidentally dropped it) versus when she willfully failed to do so (e.g. she teased the infants with it or played with it herself). Results showed that 9-, 12-, and 18-month-olds responded with more impatience (e.g. reaching, looking away, banging) when the adult was unwilling to give them the toy than when she was unable to do so. Recent research extended these results to 6-month-olds (Marsh, Stavropoulos, Nienhuis \& Legerstee, 2010). These data demonstrate that infants are sensitive to and respond appropriately in fairly complex social interactions involving incomplete goals, but it remains unclear whether infants were actually predicting the goals of the experimenter in the unwilling and unable events.

Brandone and Wellman (2009) further examined infants' understanding of successful versus unsuccessful human actions by habituating infants to an actor reaching in an arcing motion over a barrier to retrieve a ball. In some conditions the actor successfully retrieved the ball; in others, he failed to do so (Figure 1). Following habituation, infants in both conditions saw test events where the barrier was absent and the actor successfully retrieved the ball. In direct-reach events, the actor reached directly for the ball; in indirect-reach 


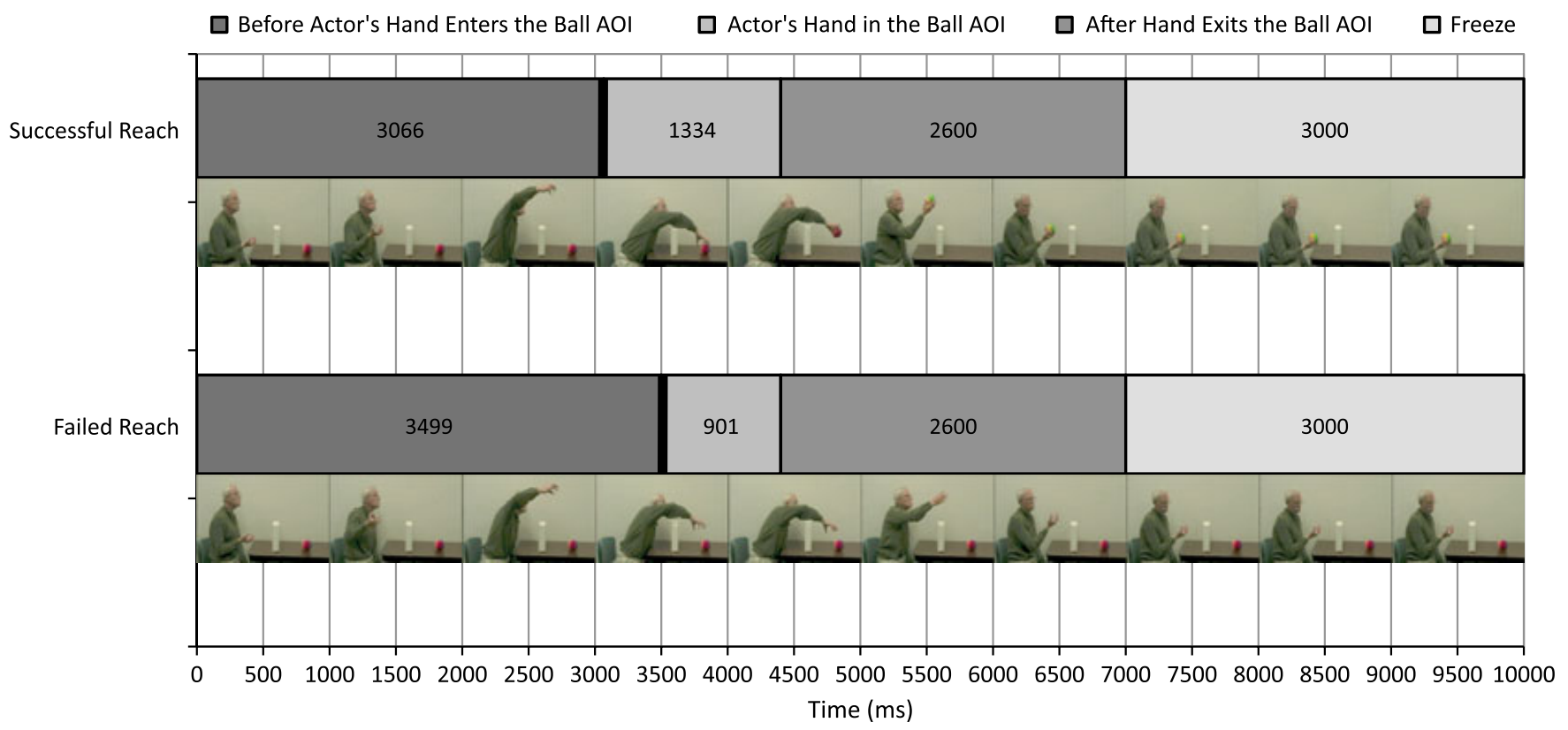

Figure 1 Depiction of the successful and failed action events. Events are divided into segments: (a) before the actor's hand enters the ball Area of Interest (AOI); (b) while the actor's hand is in the ball AOI either grasping or hovering over the ball; (c) after the actor's hand exits the ball AOI and returns to the starting position; (d) the video frozen with the actor looking down at his hand holding the ball or his empty hand. The time course of the video segments is indicated. The thick line indicates the anticipatory look cut-off for each event.

events, the actor employed the same arcing reach as in habituation. Results showed that 8-, 10-, and 12-montholds looked longer at the indirect- than the direct-reach events following habituation to a successful action (see also Phillips \& Wellman, 2005), suggesting that they encoded the successful reaching action in terms of its goal (getting the ball) and not its perceptual features (reaching in an arcing motion). After failed reaches, however, only 10- and 12-month-olds looked longer at the indirect-reach event; 8-month-olds looked equally at both events. These findings demonstrate that by 10 months of age infants interpret failed human actions in terms of their unobserved goals. However, the looking time data in this study are retrospective and thus cannot tell us whether infants are generating predictions about the actor's goal or rather responding to the action after it is completed.

One intriguing attempt to assess infants' ability to make predictions about incomplete human actions comes from Southgate, Johnson, El Karoui and Csibra (2010). They used EEG to measure neural activity in 9-month-old infants while they viewed either an incomplete action that could be interpreted as goal directed (e.g. a grasping hand reaching behind an occluder) or an incomplete action that could not be interpreted as goal directed (e.g. a grasping hand reaching in the absence of an object). The infants showed evidence of motor activation only when observing the action that could be construed as goal directed (e.g. reaching behind an occluder) and not when the action was clearly non-goal-directed, a pattern of results consistent with the finding that adults show selective activation in the motor cortex when observing goal-directed actions (Muthukumaraswamy, Johnson \& McNair, 2004). Southgate and colleagues argued that for this pattern of results to emerge, infants must have generated a prediction about the goal of the grasping hand. However, EEG data can only provide indirect evidence of prediction in the absence of any behavioral confirmation. In addition, the question of how infants understand incomplete actions differs from our question about failed actions. Therefore, questions remain regarding whether and when infants can anticipate the goals of actions that repeatedly fail to achieve their intended outcomes, and how infants respond to events in which their initial goal predictions are not confirmed.

To address these questions, in the current study we used eye tracking to examine how infants and adults process repeated, ongoing failed versus successful intentional actions. We modeled our design after Brandone and Wellman (2009). Eight-month-olds, 10-month-olds, and adults were repeatedly shown an event in which a 
man reached over a barrier and either successfully or unsuccessfully retrieved a ball (Figure 1). First, we investigated whether infants are capable of prospectively processing actions that repeatedly fail to achieve their intended outcome. To do so, we assessed whether and at what age infants would produce anticipatory looks to the intended goal of the failed reaching action. Importantly, we compared infants' ability to generate predictions about failed actions with their ability to do so for comparable successful actions. Second, we investigated how infants respond to events in which their initial goal predictions are not confirmed. To do so, we examined whether and how infants' initial goal anticipations changed across trials in response to further evidence of the actor's success or failure.

We reasoned that for both successful and failed reaching actions, action understanding would be demonstrated by anticipatory looks to the actor's intended goal (i.e. the ball). Thus, we predicted that, consistent with findings from Brandone and Wellman (2009) and previous eye-tracking research (e.g. Cannon \& Woodward, 2012; Falck-Ytter et al., 2006; Kanakogi \& Itakura, 2011), 8-month-olds, 10-month-olds and adults would produce anticipatory looks to the ball while observing the successful reaching event. However, we predicted that only 10-month-olds and adults would produce anticipatory looks in the case of the failed reaching event. Such a pattern would be consistent with data suggesting that the ability to interpret failed actions emerges later than the ability to interpret comparable successful actions (Brandone \& Wellman, 2009). In addition, we examined whether and when infants of these young ages would appropriately modify their predictions in response to accumulating evidence. In particular, in the case of the failed reaching action, we reasoned that infants who demonstrated anticipatory looks to the ball in initial trials (reflecting an understanding of the actor's intended goal) would modify their predictions in response to mounting evidence of the actor's failure to grasp the ball.

\section{Method}

\section{Participants}

Participants included 56 8- and 10-month-olds (30 males, 26 females; 8-month-olds: $n=28, M=8.41$; 10-montholds: $n=28, M=10.33$ ) and 25 undergraduates (11 males, 14 females). Participants were randomly assigned to either the successful (8-months-olds: $n=15, M=$ 8.41; 10-month-olds: $n=14, M=10.31$; undergraduates: $n=13$ ) or failed reaching condition (8-month-olds: $n=13, \quad M=8.40 ; 10$-month-olds: $n=14, \quad M=10.35$; undergraduates: $n=12$ ). An additional seven infants and five undergraduates were excluded due to fussiness $(n=1)$, poor eye-tracking signal (i.e. cases in which less than $40 \%$ of gaze acquisition attempts were successful; $n=2)$, and failure to calibrate $(n=3)$ or meet inclusion criteria (described below; $n=6$ ).

\section{Apparatus}

Eye-gaze data for infants and undergraduates were collected in separate laboratories using different Tobii eye trackers (17-inch 1750 for infants, 24-inch T60XL for undergraduates; Tobii Technology, Stockholm, Sweden). Tobii eye trackers use a corneal reflection technique to measure where participants are looking as they watch stimulus videos. Data rates for the Tobii 1750 and T60XL are $50 \mathrm{~Hz}$ and $60 \mathrm{~Hz}$, respectively. The average accuracy of both systems is in the range of 0.5 to 1 visual degree. Both of the Tobii systems introduce slight delays (verified as $50-100 \mathrm{~ms}$ ) in the relationship between the actual eye position and the estimated position. These delays have the effect of placing the estimated eye position behind the position of the moving hand, thereby somewhat reducing the likelihood of detecting anticipatory looks.

\section{Stimuli}

Participants saw one of two events identical to those in Brandone and Wellman (2009) (see Figure 1). Both events begin with a $1000 \mathrm{~ms}$ sequence in which the actor gazes over the barrier at the ball, returns to his starting position, and begins to reach. In the successful reaching event, the actor reaches over the barrier (with an arcing motion), grasps the ball, brings it back to his torso, and the video freezes. In the frozen state, the actor is holding the ball in his hand and looking down at it with a neutral expression. In the failed reaching event, the actor reaches over the barrier (with an arcing motion), but narrowly misses the ball. His hand remains separated from the ball by 2 visual degrees (i.e. roughly $2 \mathrm{~cm}$ on the screen with a viewing distance of $60 \mathrm{~cm}$ ). After hovering in this position, the actor brings his empty hand back to his torso and the video freezes. In the frozen state, the actor is looking down at his empty hand with a disappointed expression. Both reaching actions totaled $6000 \mathrm{~ms}$ in duration, followed by a $3000 \mathrm{~ms}$ freeze. This freeze duration was selected so that the total duration of the trials $(10 \mathrm{~s})$ would be comparable to the average duration of looking during habituation (9.68 s) for participants in Brandone and Wellman (2009). 
Note that due to inherent differences between successful and failed reaches, all aspects of the reaching events (e.g. rate, duration, distance) could never be equated. We elected to maintain the naturalness of the actor's reach and to equate the total duration of the reaching events (i.e. the amount of time it takes for the hand to reach for and grasp the ball in the successful event vs. reach for and hover over the ball in the failed event); however, this alignment meant that parts of the successful and failed reaches are necessarily of slightly different durations. In particular, as can be seen in Figure 1, the time before the actor's hand enters the area of interest (AOI) around the ball is $433 \mathrm{~ms}$ shorter in the successful than in the failed event because in the successful event the actor's hand needs to reach the AOI, enter it, and grasp the ball in the same amount of time that, in the failed event, the actor's hand needs to merely reach and hover at the edge of the AOI. These timing differences mean that fixations during the successful and failed reaching events occur on slightly different timescales. We account for these differences as described below (see Data processing and analysis).

\section{Procedure}

Participants sat in front of the eye tracker screen at a viewing distance of approximately $60 \mathrm{~cm}$. Infants sat on a parent's lap. Eye tracker calibration and stimulus presentation were controlled by ClearView and Tobii Studio software. The eye tracker was calibrated for each participant using a 5-point procedure. This procedure was repeated until at least three of the five points were properly calibrated for each eye (see Gredebäck, Johnson \& von Hofsten, 2010, for details of the calibration procedure). In the experimental task, participants were presented with 10 successive repetitions of either the successful or failed reaching event. Reaching events alternated with a brief animation (accompanied by music) designed to ensure participants' attention to the screen. Once participants were attending to the screen and the system was able to track both eyes, the experimenter ended the attention-getter and initiated the next event.

\section{Data processing and analysis}

All data processing was performed using custom-made analysis programs in Matlab (Mathworks Inc., Natick, MA). A circular area of interest (AOI) was defined manually around the ball. The AOI subtended approximately $1^{\circ}$ beyond the outer limit of the ball. This buffer was selected based on standards in the field (see
Gredebäck et al., 2010) and estimates of the inaccuracies inherent in the Tobii systems $\left(0.5\right.$ to $\left.1.0^{\circ}\right)$.

Trials on which a participant watched the full screen for less than $50 \%$ of the reaching action were excluded from that participant's data. Across participants, 13.6\% of trials were dropped for this reason. The number of trials dropped per subject did not differ by age group or condition ( $p$ values $>.36$ ). Participants for whom five or more trials were dropped were excluded from the final data ( $n=6$ : three infants and three adults).

On the remaining trials, gaze shifts to the ball were defined as fixations if participants fixated within the AOI for (a) five consecutive gaze data points $(100 \mathrm{~ms}$ for infants; $83 \mathrm{~ms}$ for adults) or (b) 10 non-consecutive gaze data points (200 ms for infants; $166 \mathrm{~ms}$ for adults) within a period of 15 gaze data points $(300 \mathrm{~ms}$ for infants; $249 \mathrm{~ms}$ for adults). Looks that did not meet these criteria were not classified as fixations to the ball ( $7.68 \%$ of the remaining trials).

Our focal analyses examined whether or not participants' gaze shifts to the ball were anticipatory. Anticipatory looks are typically defined as looks to the goal of the action before the action is completed (e.g. FalckYtter et al., 2006). However, in our failed reaching condition the action is never technically completed. Thus, anticipatory looks were defined here using a criterion that involves a distance between the hand and ball: looks to the AOI that occur before the actor's hand is $2^{\circ}$ away from the AOI. The benefit of using a distance criterion is that it can be applied equally to the successful and failed reaching events and, thus, can be equated across conditions. This distance was selected because (a) $2^{\circ}$ is the distance between the actor's hand and the ball at the full extension of the failed reach and thus represents the smallest distance between the ball and the hand that applies to both conditions; (b) $2^{\circ}$ represents the point at which a $1^{\circ}$ buffer around the hand meets a $1^{\circ}$ buffer around the ball and thus, even given small inaccuracies in the eye-tracking system, at this distance it is clear whether a given look is to the ball or the hand. Thus, fixations to the AOI that occurred before the actor's hand was $2^{\circ}$ away from the ball were defined as anticipatory looks (see Gredebäck et al., 2010).

Note that this definition of anticipation is very stringent because it does not account for the processing lag inherent in the oculomotor system (Canfield, Smith, Brezsnyak \& Snow, 1997). Previous researchers have defined $200 \mathrm{~ms}$ as the time needed to engage the oculomotor system (e.g. Gredebäck \& von Hofsten, 2007; Gredebäck et al., 2009) and thus gaze shifts to the target location occurring within $200 \mathrm{~ms}$ of the event's completion are commonly classified as anticipatory. This criterion is arguably less conservative and has been 
called into question recently (see Cannon, Woodward, Gredebäck, von Hofsten \& Turek, 2012, for a discussion). Nevertheless, the $200 \mathrm{~ms}$ criterion is also potentially more sensitive and likely to capture anticipatory looks - especially for young children and in the case of complex events such as failed actions. Thus, in the current study we consider both criteria in our analyses. In our initial analyses examining differences between (i.e. age, gender) and within (i.e. trial) subjects in the likelihood of producing an anticipatory look, we apply the stringent criterion of $0 \mathrm{~ms}$ and classify as anticipatory fixations to the AOI that occurred before the actor's hand was $2^{\circ}$ away from the ball. In subsequent analyses of the degree to which participants' gaze shifts were reliably anticipatory, we consider both the more and less stringent criteria ( $0 \mathrm{~ms}$ and $200 \mathrm{~ms}$, respectively). We argue that because all criteria are partly arbitrary, robust patterns of results across several criteria are more informative than use of any one alone. Moreover, reporting results using both criteria strikes a balance between the more conservative and more sensitive positions.

As mentioned previously, because we equated the duration of the total reach in both conditions there were small differences in the timing of the hand's approach to the ball in the successful and failed reaching events; the time at which the actor's hand is $2^{\circ}$ from the ball occurs $433 \mathrm{~ms}$ earlier in the successful than in the failed reaching condition. Thus, if one considers only the amount of time available between when the video begins and when either of the two anticipatory look criteria occurs, participants in the failed reaching condition have a greater opportunity to produce an anticipatory look than do participants in the successful reaching condition. Another way to think about this difference is that, for their looks to be considered anticipatory, participants in the successful reaching condition have to shift their gaze to the ball faster than those in the failed reaching condition. Note that these differences work in favor of finding evidence of anticipatory looking in the failed reaching condition because infants are given additional time to look anticipatorily; however, since the same timing differences apply for each age group, they work against the hypothesis of finding age-related differences in performance in the failed reaching condition.

\section{Results}

Effects of age (8-month-olds, 10-month-olds, undergraduates; between), gender (male, female; between), and trial (1-10; within) on the likelihood of producing an anticipatory look using the $0 \mathrm{~ms}$ criterion were evaluated through the Generalized Estimating Equations
(GEE) procedure. The GEE procedure is appropriate because it can account for the underlying binary structure of the data (producing an anticipatory look or not on a given trial), and can assess both within- and between-subject effects (Liang \& Zeger, 1986). These analyses yield Wald $\chi^{2}$ values as indicators of main effects and interactions. Significant contrasts were further explored using sequential Bonferroni corrections. The degree to which the latency of participants' gaze shifts was reliably anticipatory was also examined with single sample $t$-tests (one-tailed) comparing latencies against the stringent criterion of $0 \mathrm{~ms}$ and the less conservative criterion of $200 \mathrm{~ms}$.

Using this analytic approach, we first asked how participants initially viewed the reaching events (before they witnessed a successful or failed outcome). Second, we asked whether participants who had seen the entire action sequence (and its success or failure) on Trial 1 adjusted their looking patterns on subsequent trials. Preliminary analyses showed significant effects of trial (2-10) that were best captured by aggregating data over triads of trials (Triad 1: trials 2-4; Triad 2: trials 5-7; Triad 3: trials 8-10). Because of the timing differences between the successful and failed reaching actions, we examined the two conditions in separate analyses. None of the analyses revealed any main effects or interactions with gender; thus, they are not discussed further below.

Trial 1: How did participants view the reaching event on Trial 1 - before they witnessed a successful or failed outcome?

\section{Successful reaching condition}

We first asked how participants in the successful reaching condition viewed the reaching event on Trial 1 - before they witnessed a successful outcome. Analyses yielded only a significant main effect of age group, $\chi^{2}(2)=11.90, p=.003$ : Undergraduates were more likely to produce anticipatory looks than 8- and 10month-olds, $p<.001$ (see Figure 2). Single sample $t$-tests examining when participants looked at the AOI showed that latencies of looking to the ball in Trial 1 were anticipatory for undergraduates $(0 \mathrm{~ms}$ criterion: $t(12=$ $-1.56, p=.072 ; 200 \mathrm{~ms}$ criterion: $t(12=-2.62$, $p=.012$ ), but not for 8- or 10-month-olds (see Figure 3). Thus, even on the very first trial, undergraduates revealed some tendency to expect that the actor's movements would be directed toward the ball. In contrast, infants' gaze shifts on the first trial were more reactive in nature. Neither infant age group showed immediate, first trial expectations of goal directedness in this context. 


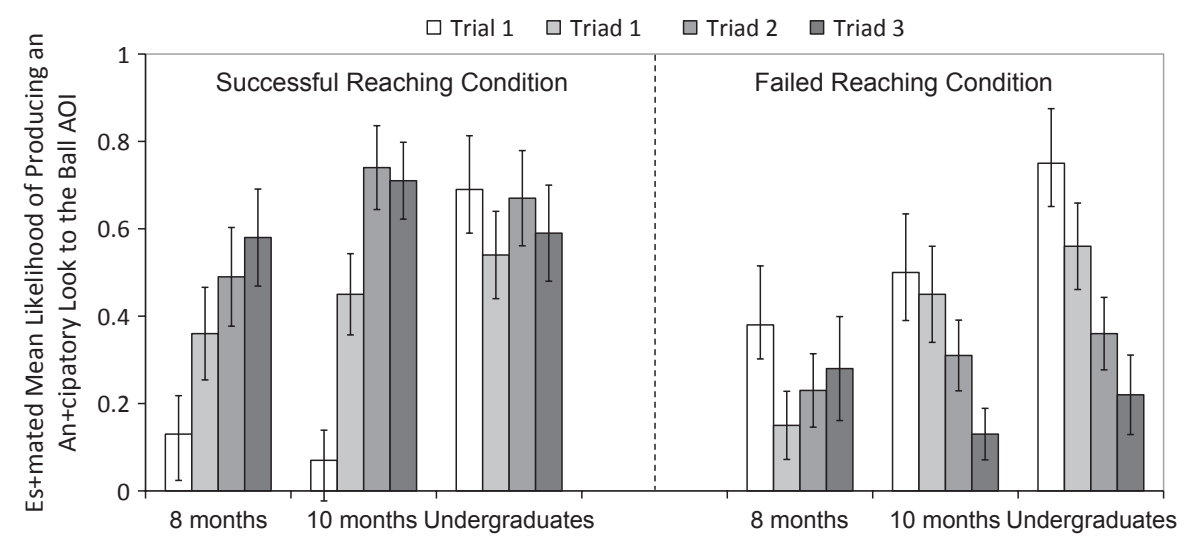

Figure 2 Estimated mean likelihood of producing an anticipatory look to the ball Area of Interest (AOI) ( \pm standard error) in the successful and failed reaching conditions as a function of trial and age group.

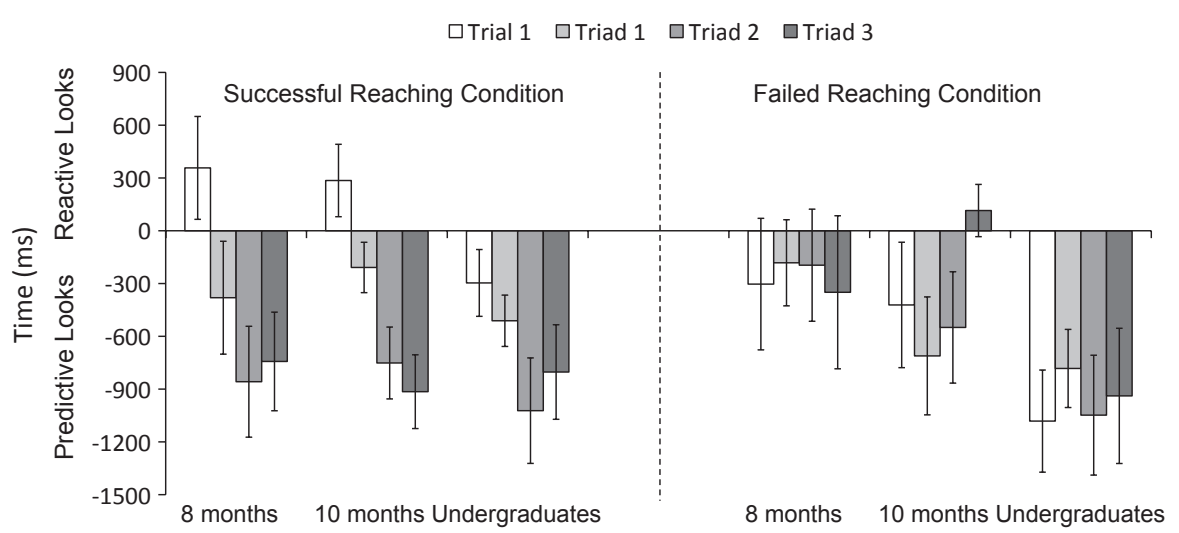

Figure 3 Mean latency of looking ( \pm standard error) to the ball Area of Interest (AOI) in the successful and failed reaching conditions as a function of trial and age group. The time at which the actor's hand enters the ball AOI is represented by the horizontal line at $0 \mathrm{~ms}$. Points below $0 \mathrm{~ms}$ indicate predictive looks; points above $0 \mathrm{~ms}$ indicate reactive looks.

Failed reaching condition

Similarly, we asked how participants in the failed reaching condition viewed the reaching event in Trial 1 - before they witnessed a failed outcome. As in the successful condition, undergraduates were more likely to produce anticipatory looks to the ball than were 8- and 10 -month-olds, $\chi^{2}(1)=3.84, p=.05$ (Figure 2). Single sample $t$-tests showed that latencies to the ball on Trial 1 were reliably anticipatory for undergraduates $(0 \mathrm{~ms}$ criterion: $t(9)=-3.73, p=.003 ; 200 \mathrm{~ms}$ criterion: $t$ $(9)=-4.42, p=.001$ ), but not for 8 - or 10-month-olds (Figure 3). Thus, in the failed reaching condition, on the very first trial undergraduates demonstrated their expectation that the actor's movements would be directed toward the ball. In contrast, infants did not show immediate expectations of goal directedness (just as they had not in the successful reaching condition).

Triads 1-3: How did participants who had seen the entire action sequence (and its success or failure) on Trial 1 adjust their looking patterns on subsequent trials?

\section{Successful reaching condition}

Did participants who had seen the entire successful action sequence on Trial 1 adjust their looking patterns on subsequent trials? Results showed a significant main effect of triad, $\chi^{2}(3)=19.04, p<.001$, and a marginal triad by age group interaction, $\chi^{2}(2)=11.51, p=.074$. Overall, there was a linear increase in the likelihood of producing an anticipatory look to the ball across triads 
$(p<.001$; see Figure 2). This effect reached significance for 8 - and 10-month-olds $(p s<.001)$ but not for undergraduates.

Single sample $t$-tests comparing latencies of looking to the AOI against the more conservative criterion of $0 \mathrm{~ms}$ confirmed that in the second and third triads, latencies to the ball were reliably anticipatory for all age groups. In Triad 1, however, although the mean latency was well below $0 \mathrm{~ms}$ for all age groups, the significance of this comparison differed by age group. Latencies to the ball in Triad 1 did not differ from $0 \mathrm{~ms}$ for 8-month-olds, were marginally anticipatory for 10 -month-olds $(p=.085)$, and were reliably anticipatory for undergraduates $(p=.003)$. When the less conservative criterion of $200 \mathrm{~ms}$ was used in the single sample $t$-test (taking into account delays in the oculomotor system), latencies to the ball were reliably anticipatory for all age groups in all triads (all $p s<.05)$. Thus, results suggest that after seeing the actor successfully complete his goal, 8-month-olds, 10-month-olds, and undergraduates increasingly produced anticipatory looks to the ball throughout the remaining successful reaching trials.

\section{Failed reaching condition}

A key question regarding infants' ability to behave flexibly in response to new evidence is how participants performed in the failed action trials after they had seen the entire failed action sequence on Trial 1. In particular, we were interested in whether infants ever generated an anticipatory look in the failed reaching condition and whether participants adjusted their looking patterns on subsequent trials as the actor repeatedly failed to meet his goal. GEE analyses on the likelihood of producing an anticipatory look showed a marginal main effect of triad, $\chi^{2}(2)=5.60, p=.061$, that is best interpreted in light of a significant triad by age group interaction, $\chi^{2}(4)=$ $11.88, p=.018$.

As can be seen in Figure 2, 10-month-olds, $\chi^{2}(1)=$ 13.31, $p<.001$, and undergraduates, $\chi^{2}(1)=5.14$, $p=.023$, showed a linear effect of triad. They were most likely to produce anticipatory looks in Triad 1. After watching the actor fail repeatedly, however, 10-montholds and undergraduates produced anticipatory looks less frequently in Triads 2 and 3. Notably, in later triads 10 -month-olds and undergraduates not only produced fewer looks to the ball that met the criterion of being anticipatory (i.e. occurring before the actor's hand entered the AOI around the ball), they also produced fewer looks to the ball overall. GEE analyses on the proportion of trials within each triad on which participants looked at the ball (anticipatorily or not) revealed a linear effect of triad, $\chi^{2}(1)=17.66, p<.001$, such that participants looked at the ball less and less across triads $\left(M_{\text {Triad } 1}=.69, M_{\text {Triad 2 }}=.50, M_{\text {Triad } 3}=.33\right)$. These results stand in contrast to those in the successful reaching condition in which participants continued to look at the ball at high levels in all triads $\left(M_{\text {Triad } 1}=.92\right.$, $M_{\text {Triad 2 }}=.90, M_{\text {Triad 1 }}=.82$ ). Overall, these findings demonstrate that 10-month-olds and undergraduates can predict the intended outcome of an action even when the action is unsuccessful and the actor repeatedly fails to achieve his goal. Moreover, 10-month-olds and adults also adjust their looking patterns in response to accumulating data regarding the actor's failure.

The pattern of results for the youngest age group in the failed reaching condition was notably different. As can be seen in Figure 2, 8-month-olds were significantly less likely to produce anticipatory looks in Triad 1 of the failed reaching events than were 10-month-olds and undergraduates, $\chi^{2}(1)=10.64, p=.002$. In subsequent trials, 8-month-olds continued to show low rates of anticipatory looks. Further analyses on the number of trials in the failed reaching condition on which participants looked at the ball overall - anticipatorily or not showed that 8-month-olds produced low levels of gaze shifts to the ball overall $\left(M_{\text {Triad 1 }}=.41, M_{\text {Triad 2 }}=.54\right.$, $\left.M_{\text {Triad 1 }}=.51\right)$. These results again stand in contrast to those for the successful reaching condition in which 8month-olds continued to look at the ball at high levels in all triads $\left(M_{\text {Triad 1 }}=.78, M_{\text {Triad 2 }}=.76, M_{\text {Triad 3 }}=.76\right)$.

Finally, for the subset of participants in the failed action condition who did look at the ball AOI on any of the nine trials after Trial 1, single sample $t$-tests examining the latency of participants' gaze shifts were conducted. Results confirmed that in Triads 1 and 2, the gaze shifts of 10-month-olds ( $0 \mathrm{~ms}$ criterion: Triad 1:t $(11)=-2.12, p=.029$ and Triad 2: $t(9)=-1.74$, $p=.058 ; 200 \mathrm{~ms}$ criterion: both $p$ s $<.05)$ and undergraduates (using both the 0 and $200 \mathrm{~ms}$ criteria, all $p s<.01)$ were reliably anticipatory. Undergraduates' gaze shifts were also reliably anticipatory in Triad 3 ( $p<.05$ using both criteria); however, 10-month-olds' were not (Figure 3). Eight-month-olds, in contrast, did not reliably predict the intended outcome of the failed action in any triad (using either the 0 or $200 \mathrm{~ms}$ criterion). Thus, when 8 -month-olds did look at the ball in the failed reaching condition, they did so late relative to the hand's arrival at the AOI.

Together these results show that by 10 (but not 8 ) months of age, infants predict the intended outcome of an action even when the action is unsuccessful and the actor repeatedly fails to achieve his goal. Further, 10month-olds and adults show evidence of adjusting their looking patterns in response to accumulating data regarding the actor's reaching failure. 


\section{Discussion}

Recent studies have suggested that during the first year of life infants can generate predictions about the goals of others' ongoing actions in situations in which the actor's goals are successfully completed and infants' initial expectations about the outcome of the action are confirmed (e.g. Cannon \& Woodward, 2012; Falck-Ytter et al., 2006; Hunnius \& Bekkering, 2010; Kanakogi \& Itakura, 2011; Kochukhova \& Gredebäck, 2010). However, mature social information processing requires the additional ability to behave flexibly and prospectively in response to more complex social events. Thus, we aimed to provide a stronger test of infants' prospective reasoning about others' actions by assessing (1) whether infants can generate predictions about actions that fail to achieve their intended outcome, and (2) how infants respond to events in which their initial goal predictions are not confirmed. We compared infants' ability to generate predictions about actions that successfully achieve their goals as well as the special and more complex case of those that repeatedly fail to do so. Results yielded three main conclusions.

First, the results confirm that infants in the first year of life show a prospective understanding of successful human actions: when observing an actor engaging in a successful goal-directed reaching action, 8- and 10month-olds produced reliably anticipatory looks to the goal of the actor's reach. These findings are consistent with those of previous studies (Cannon \& Woodward, 2012; Hunnius \& Bekkering, 2010; Kanakogi \& Itakura, 2011; Kochukhova \& Gredebäck, 2010) documenting the ability to generate predictions about ongoing successful actions in infants under 1 year of age.

Second, our data indicate that 10- (but not 8-)montholds are also capable of prospectively processing intentional actions in which the action is unsuccessful and the actor repeatedly fails to achieve his goal. When observing an actor engaging in a failed goal-directed reaching action, 10-month-olds produced reliably anticipatory looks to the goal of the actor's reach. They did so consistently in several complementary analyses using multiple criteria for what constitutes an anticipatory look. Younger infants, however, did not show this ability even using our least stringent criterion. When the actor failed to achieve his goal, 8-month-olds tracked the actor's reach in a reactive manner - looking later and sometimes not at all at the goal of the failed reach. These data add to the recent literature (e.g. Brandone \& Wellman, 2009; Hamlin et al., 2009) and provide the most compelling evidence of failed action understanding to date by demonstrating not only that 10 -month-olds evaluate failed actions retrospectively (as indicated by previous looking time research), but also that they can predict the goal of another's action prospectively after observing a failed attempt.

Importantly, the current study further demonstrates how infants use new evidence to modify their expectations about a human action. First, 8- and 10-month-olds did not show evidence of anticipatory looking until after observing the actor's first reaching action (i.e. not on Trial 1) even for successful reaches. This pattern suggests that infants used information acquired when viewing a successful or unsuccessful first reach to establish their predictions about subsequent events. In addition, 10month-olds and adults also showed evidence of modifying their looking patterns in the failed reaching condition when their initial goal predictions were not confirmed. That is, although they produced anticipatory looks to the intended outcome of the failed action in Triad 1, these looking patterns changed after watching the actor repeatedly fail to achieve his goal. These data suggest that, at least in the failed condition, participants reevaluated their expectations about the action and the actor's goal on the basis of accumulating evidence. Although learning effects have been documented in some prior studies examining infants' action prediction abilities (e.g. Gredebäck \& Melinder, 2010; Henrichs, Elsner, Elsner \& Gredebäck, 2012; Kochukhova \& Gredebäck, 2010), learning is typically observed through infants producing faster anticipations across trials (as occurred in the successful reaching condition in the current study; see Figure 3). The learning effects observed in response to the failed reach, however, are distinct. Ten-month-olds and adults in the failed reaching condition did not simply produce the same gaze shifts faster over time; rather, they qualitatively changed the pattern of their gaze shifts re-evaluating their expectations about the observed action and fixating less and less on the ball across trials. This ability to revise their expectations online fits with a model of infants as 'rational learners' who integrate prior beliefs, knowledge, and expectations with new evidence provided by the environment (Xu \& Kushnir, 2013). Moreover this ability enables infants to generate predictions about and learn from more complex social situations.

A key question arising from the current data is what explains the difference in infants' performance between 8 and 10 months of age. In particular, although 8-montholds demonstrated the ability to generate predictions about the successful reaching event, unlike 10-montholds and adults, 8-month-olds did not show evidence of anticipating the intended goal of the failed reach (see also Brandone \& Wellman, 2009) or modifying their predictions in response to the actor's repeated failure. Several interpretations are plausible. 
As one possibility, consider the essential difference between successful and failed actions. In successful actions, the actor's goal is apparent in the achieved outcome (e.g. successfully grasping the ball) whereas in failed actions the actor's goal is unrealized and thus not apparent in the action itself. That 8-month-olds in the current study only showed anticipatory looking after seeing the actor successfully grasp and retrieve the ball may suggest that at this age infants need outcome information to determine the goal of the action. This pattern of results is consistent with the view that infants' initial understandings of human action are somewhat superficial in nature: they capture certain regularities of action attested in their past experience (e.g. that actions are directed toward objects and are rational and efficient; Csibra, Gergely, Biro, Koos \& Brockbank, 1999; Gergely et al., 1995; Phillips \& Wellman, 2005; Woodward, 1998), but not the goals that motivate them. In contrast, 10-month-olds showed anticipatory looking even without seeing the actor make contact with the ball and responded appropriately in the face of accumulating evidence - suggesting that by this age infants can infer an actor's goal prospectively and use that goal information flexibly to make online predictions (see also Cannon \& Woodward, 2012). One intriguing possibility, and one we favor, is that older infants are able to do this because they have begun to view human behavior as motivated by the subjective internal states of others.

Alternatively, 8- and 10-month-olds may perform differently in the failed reaching condition as a result of varying expectations gleaned from experience engaging in or observing intentional actions. Gredebäck and colleagues (2009) propose that action understanding and, specifically, goal anticipation are mediated by a process of direct matching in which an observed action is mapped onto a motor representation of that action (e.g. see also Rizzolatti, Fogassi \& Gallese, 2001). On this view, an observer's own action capabilities, not the observer's understanding of the actor's goal, mediate goal anticipation. Support for this position comes from research documenting correlations between infants' own action abilities and their goal anticipations (e.g. Cannon et al., 2012; Falck-Ytter et al., 2006; Gredebäck \& Kochukhova, 2010). This interpretation could also help to explain the age effects observed in the current study. In particular, there may be developmental differences in infants' ability to reach around barriers or engage in goal-directed actions more broadly (Piaget, 1953; Willatts, 1999) that are related to infants' ability to interpret and make predictions about the reaching actions presented here.

It is also possible that 8- and 10-month-olds performed differently as a result of differing levels of experience observing others' actions and, in particular, others' reaching hands (see Sommerville, Woodward \& Needham, 2005, and Biro \& Leslie, 2007, for related discussions). Older infants and adults may have a stronger expectation than 8-month-olds that reaching hands tend to contact objects toward which they approach. Note, however, that on both these accounts it is unclear why 8-month-olds' performance should differ across the successful and failed reaching conditions because both conditions used the same simple action. Nevertheless, developmental differences in infants' own experience as intentional agents or expectations about hands and their likely actions may help to explain the age effects observed here.

A final possibility to consider is that processing limitations, slower computation abilities, or domaingeneral differences in the capacity to generate predictions and consider evidence may have prevented the youngest infants from demonstrating their prospective understanding of the failed reaching action or modifying their expectations in response to accumulating evidence. Note that 8-month-olds performed equivalently to older infants and adults in the successful reaching condition, where because of small timing differences their anticipatory looks had to be slightly faster than needed in the failed reaching condition; therefore, any performancelevel explanation would need to account for why 8-month-olds demonstrate anticipation abilities in the successful condition and not in the failed condition. Nevertheless, caution is warranted in interpreting 8-month-olds' inability to generate predictions about complex unsuccessful actions and flexibly modify their goal predictions. Further research is needed to examine these and other explanations for changes in action understanding and prediction during infancy.

In sum, the current data expand our understanding of infants' sophisticated social cognitive abilities. Our findings add to the growing literature on infants' ability to interpret and generate predictions about the goals underlying other people's actions. Results show that by 10 months of age, infants can generate predictions about actions that fail to achieve their intended outcome. Furthermore, infants can re-evaluate their expectations about others' actions on the basis of accumulating evidence. This flexible, prospective social information processing ability likely plays a crucial role in how infants interact with and learn from others.

\section{Acknowledgements}

This research was supported by an NSF Graduate Research Fellowship and Lehigh University funds to Brandone, a grant from the National Institute of Health 
(HD022149) to Wellman, and grants from the National Institute of Health (HD-37082) and the J.S. McDonnell Foundation (220020096) to Aslin. Portions of these data were submitted in partial fulfillment of the requirements for a doctoral degree at the University of Michigan. We thank Susan Gelman, Chelsea Heatherington, Alyssa Thatcher, Johnny Wen, and our participants.

\section{References}

Baldwin, D.A., Baird, J.A., Saylor, M.M., \& Clark, M.A. (2001). Infants parse dynamic action. Child Development, $\mathbf{7 2}$, 708-717.

Baldwin, D.A., \& Moses, L.J. (2001). Links between social understanding and early word learning: challenges to current accounts. Social Development, 10, 309-329.

Behne, T., Carpenter, M., Call, J., \& Tomasello, M. (2005). Unwilling versus unable: infants' understanding of intentional action. Developmental Psychology, 41 (2), 328-337.

Biro, S., \& Leslie, A. (2007). Infants' perception of goaldirected actions: development through cues-based bootstrapping. Developmental Science, 10, 379-398.

Brandone, A.C., \& Wellman, H.M. (2009). You can't always get what you want: infants understand failed goal-directed actions. Psychological Science, 20, 85-90.

Canfield, R.L., Smith, E.G., Brezsnyak, M.P., \& Snow, K.L. (1997). Information processing through the first year of life. Monographs of the Society for Research in Child Development, 62 (2, Serial No. 250)

Cannon, E.N., \& Woodward, A.L. (2012). Infants generate goal-based action predictions. Developmental Science, 15, 292-298.

Cannon, E.N., Woodward, A.L., Gredebäck, G., von Hofsten, C., \& Turek, C. (2012) Action production influences 12-month-old infants' attention to others' actions. Developmental Science, 15, 35-42.

Csibra, G., \& Gergely, G. (2007). 'Obsessed with goals': functions and mechanisms of teleological interpretation of actions in humans. Acta Psychologica, 124, 60-78.

Csibra, G., Gergely, G., Biro, S., Koos, O., \& Brockbank, M. (1999). Goal attribution without agency cues: the perception of 'pure reason' in infancy. Cognition, 72, 237-267.

Daum, M., Prinz, W., \& Aschersleben, G. (2008). Encoding the goal of an object-directed but uncomplete reaching act in 6- and 9-month-old infants. Developmental Science, 11, 607619.

Falck-Ytter, T., Gredebäck, G., \& von Hofsten, C. (2006). Infants predict other people's action goals. Nature Neuroscience, 9 (7), 878-879.

Gergely, G., Nadasdy, Z., Csibra, G., \& Biro, S. (1995). Taking the intentional stance at 12 months of age. Cognition, 56, 165-193.

Gredebäck, G., Johnson, S., \& von Hofsten, C. (2010). Eye tracking in infancy research. Developmental Neuropsychology, 35, 1-19.
Gredebäck, G., \& Kochukhova, O. (2010). Goal anticipation during action observation is influenced by synonymous action capabilities: a puzzling developmental study. Experimental Brain Research, 202, 493-497.

Gredebäck, G., \& Melinder, A.M.D. (2010). Infants' understanding of everyday social interactions: a dual process account. Cognition, 114, 197-120.

Gredebäck, G., Stasieweicz, D., Falck-Ytter, T., Rosander, K., \& von Hofsten, C. (2009). Action type and goal type modulate goal-directed gaze shifts in 14-month-olds. Developmental Psychology, 45, 1190-1194.

Gredebäck, G., \& von Hofsten, C. (2007). Taking an action perspective on infant's object representations. Progress in Brain Research, 164, 265-282.

Hamlin, J.K., Hallinan, E.V., \& Woodward, A.L. (2009). Do as I do: 7-month-old infants selectively reproduce others' goals. Developmental Science, 11, 487-494.

Hamlin, J.K., Newman, G.E., \& Wynn, K. (2009). Eight-monthold infants infer unfulfilled goals, despite ambiguous physical evidence. Infancy, 14, 579-590.

Henrichs, I., Elsner, C., Elsner, B., \& Gredebäck, G. (2012). Goal salience affects infants' goal-directed gaze shifts. Frontiers in Psychology, 3, 391.

Hunnius, S., \& Bekkering, H. (2010). The early development of object knowledge: a study on infants' visual anticipations during action observation. Developmental Psychology, 46, 446-454.

Kanakogi, Y., \& Itakura, S. (2011). Developmental correspondence between action prediction and motor ability in early infancy. Nature Communications, 2, 341.

Kochukhova, O., \& Gredebäck, G. (2010). Preverbal infants anticipate that food will be brought to the mouth: an eye tracking study of manual feeding and flying spoons. Child Development, 81, 1729-1738.

Legerstee, M., \& Markova, G. (2008). Variation in 10-month-old imitation of people and things. Infant Behavior and Development, 31, 81-91.

Liang, K.Y., \& Zeger, S.L. (1986). Longitudinal data analysis using generalized linear models. Biometrika, 73, 13-22.

Marsh, H.L., Stavropoulos, J., Nienhuis, T., \& Legerstee, M. (2010). Six- and 9-month-old infants discrinimate between goals despite similar action patterns. Infancy, 15, 94-106.

Muthukumaraswamy, S.D., Johnson, B.W., \& McNair, N.A. (2004). Mu rhythm modulation during observation of an object-directed grasp. Cognitive Brain Research, 19, 195-201.

Phillips, A.T., \& Wellman, H.M. (2005). Infants' understanding of object-directed action. Cognition, 98, 137-155.

Piaget, J. (1953). The origins of intelligence in the child. London: Routledge \& Kegan Paul.

Rizzolatti, G., Fogassi, L., \& Gallese, V. (2001). Neurophysiological mechanisms underlying the understanding and imitation of action. Nature Reviews Neuroscience, 2, 661-670.

Senju, A., Southgate, V., Snape, C., Leonard, M., \& Csibra, G. (2011). Do 18-month-olds really attribute mental states to others? A critical test. Psychological Science, 22 (7), 878-880.

Sommerville, J.A., Woodward, A.L., \& Needham, A. (2005). Action experience alters 3-month-old infants' perception of others' actions. Cognition, 96, B1-11. 
Southgate, V., \& Csibra, G. (2009). Inferring the outcome of an ongoing novel action at 13 months. Developmental Psychology, 45, 1794-1798.

Southgate, V., Johnson, M.H., El Karoui, I., \& Csibra, G. (2010). Motor system activation reveals infants' online prediction of others' goals. Psychological Science, 21, 355-359.

Southgate, V., Senju, A., \& Csibra, G. (2007). Action anticipation through attribution of false belief by 2-year-olds. Psychological Science, 18, 587-592.

Tomasello, M. (1999). The cultural origins of human cognition. Cambridge, MA: Harvard University Press.
Willatts, P. (1999). Development of means-end behavior in young infants: pulling a support to retrieve a distant object. Developmental Psychology, 35, 651-667.

Woodward, A.L. (1998). Infants selectively encode the goal object of an actor's reach. Cognition, 69, 1-34.

Xu, F., \& Kushnir, T. (2013). Infants are rational constructivist learners. Current Directions in Psychological Science, 22, $28-32$.

Received: 20 May 2011

Accepted: 9 May 2013 